\title{
Opportunities for diagnosing cytomegalovirus in pulmonary infections
}

Cytomegalovirus (CMV) infection is considered a major cause of morbidity and mortality in organ and bone marrow transplant recipients and patients with the acquired immune deficiency syndrome (AIDS). Untreated CMV pneumonia carries a mortality approaching $100 \%$. Other syndromes may include fever with or without lymphocytosis, retinitis, encephalitis, duodenitis, and colitis, although only the first satisfies strict criteria for a causal association with the virus. ${ }^{1}$

Two hypotheses have been proposed to explain the pathogenesis of CMV pneumonia. Either pre-existing lung damage and immunodeficiency reduce the magnitude of virus-induced tissue destruction required to produce pneumonia, ${ }^{2}$ or the pneumonia reflects $T$ cell-mediated immune reactivity directed against CMV antigens. ${ }^{3}$ The first predicts a preventative role for avoidance of iatrogenic immunosuppression or pulmonary toxins, whereas the second is compatible with a therapeutic role for CMV immunoglobulin. The severity of CMV pneumonia in thoracic allograft recipients which led to the study reported by Egan and colleagues on pages $9-13$ of this issue of Thorax is explained either by immunosuppression and pre-existing lung damage ${ }^{2}$ or by $\mathrm{T}$ cell immunopathology associated with lung rejection. ${ }^{3}$

Many strategies may limit the clinical impact of CMV pneumonia. Anti-CMV chemotherapy with ganciclovir or foscarnet are often ineffective in established CMV pneumonia. ${ }^{5}$ Adjunctive CMV immunoglobulin therapy was suggested to block $\mathrm{T}$ cell immunoreactivity against $\mathrm{CMV}$ antigens, but the pathogenic role of such immunopathology ${ }^{2}$ and the efficacy of immunotherapy remain unproven. ${ }^{6}$ Prophylactic high dose acyclovir is safe and possibly effective. ${ }^{7}$ Pre-emptive ganciclovir treatment when pulmonary CMV infection is detected by bronchoalveolar lavage has been shown to be efficacious. ${ }^{8}$ Nonetheless, the potential benefit of prophylactic ganciclovir for all immunosuppressed patients must be balanced against the possible emergence of pathogenic drug-resistant virus. ${ }^{9}$ Both ganciclovir and foscarnet have major adverse effects - bone marrow suppression and nephrotoxicity, respectively - and the synergistic myelotoxicity of ganciclovir and zidovudine largely preclude their combined administration. ${ }^{10}$ Prophylactic CMV immunoglobulin may have a role in high risk CMV seronegative organ transplant recipients whose donors are seropositive. ${ }^{11}$

Preferential matching of organ transplant recipients and donors for either CMV antibody status or HLA antigens has been proposed, the former to avoid the high risk CMV positive donor, CMV negative recipient combination, ${ }^{12}$ and the latter to minimise graft rejection and hence severe CMV disease associated with immunosuppressive antirejection treatment. ${ }^{13}$ Graft survival is improved by HLA matching in renal and cardiac transplant recipients, ${ }^{14}{ }^{15}$ and graft rejection could be reduced by DR antigen matching in heart transplant recipients. ${ }^{16}$ Increased graft rejection consequent on CMV matching at the expense of HLA mismatching could therefore lead to increased iatrogenic immunosuppression. The rapid latex agglutination assay used to determine donor and recipient CMV antibody status immediately before transplantation is prone to produce occasional false negative ${ }^{17}$ and false positive results. ${ }^{18}$ This might lead to inadvertent matching of seronegative re- cipients with seropositive donors if the test on the recipient or donor gave a falsely positive or a falsely negative result, respectively. Moreover, iatrogenic immunosuppression is a crucial component of the severity of any CMV disease. ${ }^{19}$ The combination of erroneous CMV matching producing a high risk $C M V$ seronegative recipient $C M V$ seropositive donor pairing and marked immunosuppression consequent on HLA mismatching is therefore likely to be a lethal concoction. In addition, CMV matching at the expense of HLA matching will only reduce the clinical impact of CMV disease if severe manifestations are largely confined to seronegative recipients of organs from seropositive donors. However, local experience indicates that fatal CMV pneumonia can occur in previously seropositive renal transplant recipients given intensive antirejection treatment.

HLA matching appears to be associated with a low incidence of graft loss and severe CMV disease in renal allograft recipients, presumably because iatrogenic immunosuppression is thereby minimised..$^{131419}$ At present, heart, heart-lung, and lung allograft recipients and their donors are largely matched for CMV antibody status rather than HLA antigens. ${ }^{12}$ Prospective HLA matching in thoracic transplantation should improve its success ${ }^{16}$ and also minimise severe CMV disease. ${ }^{13}$

Progress in the rapid diagnosis of CMV disease followed the development of techniques for the early detection of virus replication in cell cultures 24-48 hours after inoculation. ${ }^{20} \mathrm{~A}$ viral cytopathic effect visible by light microscopy and indicative of a positive virus isolation result only appears after 1-4 weeks. CMV immediate early or early antigen detection in cell culture by immunofluorescence using specific murine monoclonal antibodies (also known as detection of early antigen fluorescent foci) ${ }^{21}$ is widely available and provides early diagnosis of active and, in particular, pulmonary CMV infection by respective examination of urine or bronchoalveolar lavage fluid..$^{82022}$ Most patients with pulmonary CMV infection either have or will shortly develop CMV pneumonia and should be treated promptly with ganciclovir. Immediate early antigen detection is not as sensitive as conventional cell culture. ${ }^{20}$ Therefore, if CMV pneumonia is diagnosed, treatment should be continued for a minimum of 14 days. If the rapid test for CMV is negative, treatment started on empirical grounds before the immediate early antigen result is available may be discontinued only if CMV pneumonia is excluded on clinical grounds.

The incomplete effectiveness of treatment of CMV pneumonia, suboptimal performance of available rapid diagnostic tests, and continuing high incidence of CMV disease (particularly pneumonia) in thoracic transplant recipients prompted Egan et al to initiate prospective surveillance for CMV infection and disease in their patients. ${ }^{4}$ In this issue of Thorax they assess the diagnostic and prognostic significance of quantitation of $\mathrm{CMV}$ antigenaemia. Peripheral blood polymorphonuclear neutrophil leucocytes positive for viral lower matrix protein were enumerated in cytospin preparations. High level antigenaemia ( $>50$ antigen positive cells per $2 \times 10^{5}$ neutrophils) was detected 2-44 (median 14) days before onset of CMV disease in five patients (four with pneumonia, one with duodenitis), but six patients who remained disease-free had similar antigenaemia giving a positive predictive value of only $46 \%$. The negative predictive value was $100 \%$. 
An alternative approach to the early identification of patients who will subsequently develop CMV disease is the assay of CMV viraemia. Blood anticoagulated with preservative-free heparin is inoculated on human fibroblast cell monolayers, and immediate early antigen detection is performed 24-48 hours later. ${ }^{21}$ In comparison with quantitation of CMV antigenaemia, this approach has a number of disadvantages. It depends on the production and maintenance of fastidious human cell lines which may show toxic effects or succumb to bacterial or fungal overgrowth following specimen inoculation. ${ }^{20}$ Cell toxicity appears to be a significant problem with blood specimens such that, in one recent study, only one of 15 specimens positive in cell culture gave a positive result in the rapid test. ${ }^{23}$ Heparinised blood samples probably only yield positive results when high level viraemia is present, as heparin is a potent inhibitor of CMV replication.$^{24}$ However, the cut-off for a positive result is likely to be more variable than when CMV antigenaemia is quantitated. With increasing time for specimen transport from patient to laboratory, heparin increasingly inhibits CMV infectivity. ${ }^{24}$ In contrast, cytolysis of neutrophils is unlikely to selectively affect CMV antigen positive cells and so the relative level of antigenaemia should be unaltered. Nonetheless, CMV viraemia and antigenaemia assays share relatively low positive predictive values. ${ }^{423}$ Use of the polymerase chain reaction (PCR) rather than immediate early antigen detection to monitor CMV viraemia may be confounded by the ultrasensitivity of the PCR which can detect viral DNA in peripheral blood mononuclear cells from healthy persons. ${ }^{25}$

Before introducing regular surveillance for CMV antigenaemia in their immunosuppressed transplant or AIDS patients, clinicians should balance the advantages of early identification of patients likely to progress to severe CMV disease against the logistic problems involved and the lack of cost-benefit analyses. Specimens must be tested in the laboratory within 3-5 hours of collection, ${ }^{4}$ thus requiring a dedicated transport system. The requirement for cytocentrifugation poses safety problems, particularly if specimens from patients with AIDS are processed, because aerosol generation is inevitable. The work should therefore be confined within a microbiological safety cabinet. All these features will render CMV antigenaemia testing an expensive procedure. However, if specimens are only collected for diagnosis in symptomatic patients, CMV antigenaemia assays are an attractive alternative to immediate early antigen detection.

The considerable cost of regular surveillance for high level CMV antigenaemia might be justifiable if, when all patients with a positive result were treated pre-emptively with ganciclovir or foscarnet, CMV associated mortality or morbidity was reduced. In particular, a consequential reduction in the costs of hospitalisation, drugs, and other care would be persuasive evidence. The low positive predictive value and high incidence of high level CMV antigenaemia in the study reported by Egan et $a l^{4}$ might be seen to indicate that a cost-benefit analysis would not favour the introduction of antigenaemia testing. Only 21 of 32 thoracic transplant recipients $(66 \%)$ remained free of high level antigenaemia and therefore might have been considered not eligible for pre-emptive antiviral chemotherapy. At least half the patients selected to receive preemptive treatment on the basis of a possible screening test were not destined to develop CMV disease. As CMV antigenaemia preceded CMV disease by 2-44 days, any pre-emptive antiviral treatment might either be too late or have to be given for at least six weeks. However, the costs of inpatient care on a high dependency unit for transplant patients will be high, and therefore the outcome of a costbenefit analysis cannot be predicted.

In conclusion, assay of CMV antigenaemia rather than viraemia is probably the preferred option for prediction of CMV disease if surveillance for pulmonary CMV infection is considered too invasive in immunosuppressed patients at high risk of serious $\mathrm{CMV}$ disease. Alternative strategies for the prevention of CMV associated morbidity and mortality, such as improved HLA matching in solid organ transplantation, high dose acyclovir prophylaxis for bone marrow (and perhaps other) transplant recipients, ${ }^{26}$ and selective CMV immunoglobulin prophylaxis, should nonetheless be pursued. The only option in patients with AIDS appears to be early antiviral therapy as soon as CMV disease is diagnosed. Surveillance for CMV antigenaemia may currently be justified in thoracic transplant recipients, given the high incidence of serious CMV disease. Such surveillance has not, however, been subjected to costbenefit analysis and may merely be an expensive luxury.

Division of Virology,

DAVID J MORRIS

Department of Pathological Sciences,

Medical School (Stopford Building),

Oxford Road,

Manchester M13 9PT,

$U K$.

1 Morris DJ. Epidemiological evidence is crucial as proof of causation in cytomegalovirus disease. $\mathcal{F}$ Infect 1991;23:233-40.

2 Morris DJ. Cytomegalovirus pneumonia - a consequence of immunosuppression and pre-existing lung damage rather than immunopathology? Respir Med 1993;87:345-9.

3 Grundy JE, Shanley JD, Griffiths PD. Is cytomegalovirus pneumonia in transplant patients an immunopathological condition? Lancet 1987; ii: 996-9.

4 Egan JJ, Barber L, Lomax J, Fox A, Yonan N, Rahman AN, et al. Detection of human cytomegalovirus antigenaemia: a rapid diagnostic technique for predicting cytomegalovirus infection/pneumonitis in lung and heart transplant recipients. Thorax 1995;50: 9-13.

5 Morris DJ. Antiviral chemotherapy for cytomegalovirus disease. 7 Antimicrob Chemother 1988;21:519-24.

6 Morris DJ. Ganciclovir and immune globulin in cytomegalovirus pneumonia. Ann Intern Med 1989;110:575.

7 Morris DJ. Antiviral chemoprophylaxis for cytomegalovirus infection. $\mathcal{F}$ Antimicrob Chemother 1988;22:569.

8 Schmidt GM, Horak DA, Niland JC, Duncan SR, Forman SJ, Zaia JA, et al. A randomised controlled trial of prophylactic ganciclovir for cytomegalovirus pulmonary infection in recipients of allogenic bone marrow transplants. N Engl f Med 1992;324:1005-11.

9 Erice A, Chou S, Biron KK, Stanat SC, Balfour HH Jr, Jordan MC. Progressive disease due to ganciclovir-resistant cytomegalovirus in immunocompromised patients $N$ Engl $¥$ Med 1989;320:289-93.

10 Morris DJ. Adverse effects and drug interactions of clinical importance with antiviral drugs. Drug Safety 1994;10:281-91.

11 Morris DJ. Therapeutic and prophylactic efficacy of cytomegalovirus immune globulin. Serodiagn Immunother 1988;2:387-9.

12 Williams PF, Wreghitt T, Joysey V, Evans DB, Calne RY. Cytomegalovirus matching in renal transplantation. Lancet 1988;ii:569.

13 Morris DJ, Martin S, Dyer PA, Hunt L, Mallick NP, Johnson RWG. HLA mismatching and cytomegalovirus infection as risk factors for transplant failure in cyclosporin-treated renal allograft recipients. F Med Virol 1993; 41:324-7.

14 Dyer PA, Johnson RWG, Martin S, Bakran A, Gokal R, Harris R, et al. Evidence that matching for HLA antigens significantly increases transplant survival in 1001 renal transplants performed in the North West region of England. Transplantation 1989;48:131-5.

15 Khaghani A, Yacoub M, McCloskey D, Awad J, Burden M, Fitzgerald M, et al. The influence of HLA matching, donor/recipient sex, and incidence of acute rejection on survival in cardiac transplant recipients receiving cyclosporin A and azathioprine. Transplant Proc 1989;21:798-800.

16 Sheldon S, Hasleton PS, Yonan NA, Rahman AN, Campbell CS, Deiraniya AK, et al. Rejection in heart transplantation strongly correlates with HLADR antigen mismatch. Transplantation 1994;58:1-4.

17 Choo S, Scott KM. Latex agglutination and enzyme immunosorbent assays for cytomegalovirus serologic testing of transplant donors and recipients. fClin Microbiol 1988;26:2116-9.

18 Morris DJ, Klapper PE, Crosdale E, White J. Screening of bone marrow and kidney transplant donors and recipients for cytomegalovirus antibody by commercial latex agglutination or enzyme-linked immunosorbent assays. F Virol Methods 1990;30:339-42.

19 Morris DJ, Longson M, Postlethwaite RJ, Mallick NP, Johnson RWG. Donor seropositivity and prednisolone therapy as risk factors for cytomegalovirus infection and disease in cyclosporin-treated renal allograft recipients. $Q \mathcal{F}$ infection and disease in

20 Morris DJ, Lomax J, Craske J, Longson M, Fox AJ. Effect of centrifugal enhancement of infectivity on the rapid detection of human cytoenhancement of infectivity on the rapid detection of human cytomegalovirus in cell culture by immunofluorescence using a monoclonal
antibody to an immediate early nuclear antigen. Serodiagn Immunother antibody to an

21 Griffiths PD, Panjwani DD, Stirk PR, Ball MG, Ganczakowski M, Blacklock 
$\mathrm{HA}$, et al. Rapid diagnosis of cytomegalovirus infection in immunocompromised patients by detection of early antigen fluorescent foci. Lancet 1984;ii:1242-4.

22 Morris DJ, Longson M. Laboratory diagnosis of simultaneous cytomegalovirus and herpes simplex virus infection. I Infect 1988;16:309-10.

23 Kidd IM, Fox JC, Pillay D, Charman H, Griffiths PD, Emery VC. Provision of prognostic information in immunocompromised patients by routine
application of the polymerase chain reaction for cytomegalovirus. Transapplication of the polymerase
plantation 1993;56:867-71.
24 Kimpton CP, Corbitt G, Morris DJ. Inhibitory effects of various anticoagulants on the infectivity of human cytomegalovirus. $f$ Virol Methods 1989;24:335-46.

25 Morris DJ, Kimpton CP, Corbitt G. Persistence of cytomegalovirus in peripheral blood from blood donors. BMF 1989;299:1164-5.

26 Prentice HG, Gluckman E, Powles RL, Ljungman P, Milpied NJ, Ranada JMF, et al. Impact of long term acyclovir on cytomegalovirus infection and survival after allogeneic bone marrow transplantation. Lancet 1994; 343:749-53. 